\title{
Effect of GABRA2 Genotype on Development of Incentive- Motivation Circuitry in a Sample Enriched for Alcoholism Risk
}

\author{
Mary M Heitzeg*,, ,2, Sandra Villafuerte ${ }^{1,3}$, Barbara J Weiland ${ }^{1,2,4}$, Mary-Anne Enoch ${ }^{5}$, Margit Burmeister ${ }^{1,3,6}$, \\ Jon-Kar Zubieta ${ }^{1,3}$ and Robert A Zucker ${ }^{1,2}$ \\ 'Department of Psychiatry, University of Michigan, Ann Arbor, MI, USA; ${ }^{2}$ Addiction Research Center, University of Michigan, Ann Arbor, MI, USA; \\ ${ }^{3}$ Molecular and Behavioral Neuroscience Institute, University of Michigan, Ann Arbor, MI, USA; ${ }^{4}$ Department of Psychology and Neuroscience, \\ University of Colorado Boulder, Boulder, CO, USA; ${ }^{5}$ Laboratory of Neurogenetics, National Institute on Alcohol Abuse and Alcoholism, NIH, \\ Bethesda, MD, USA; ${ }^{6}$ Department of Human Genetics, University of Michigan, Ann Arbor, MI, USA
}

\begin{abstract}
Heightened reactivity of the incentive-motivation system has been proposed to underlie adolescent-typical risky behaviors, including problem alcohol involvement. However, even in adolescence considerable individual variation in these behaviors exists, which may have genetic underpinnings and be related to variations in risk for later alcohol use disorder (AUD). Variants in GABRA2 have been associated with adult alcohol dependence as well as phenotypic precursors, including impulsiveness and externalizing behaviors. We investigated the impact of GABRA2 on the developmental trajectory of nucleus accumbens (NAcc) activation during anticipation of monetary reward from childhood to young adulthood. Functional MRI during a monetary incentive delay task was collected in 175 participants, with the majority $(n=151)$ undergoing repeated scanning at I- to 2 -year intervals. One group entered the study at age $8-13$ years $(n=76)$ and another entered at age 18-23 years $(n=99)$. Most participants were children of alcoholics $(79 \%)$ and thus at heightened risk for AUD. A total of 473 sessions were completed, covering ages 8-27 years. NAcc activation was heightened during adolescence compared with childhood and young adulthood. GABRA2 genotype (SNP rs279858) was associated with individual differences in NAcc activation specifically during adolescence, with the minor allele $(\mathrm{G})$ associated with greater activation. Furthermore, NAcc activation mediated an effect of genotype on alcohol problems $(n=104)$. This work demonstrates an impact of GABRA2 genotype on incentive-motivation neurocircuitry in adolescence, with implications for vulnerability to alcoholism. These findings represent an important step toward understanding the genetic and neural basis of individual differences in how risk for addiction unfolds across development.

Neuropsychopharmacology (20I4) 39, 3077-3086; doi:I0.1038/npp.20 I4.16I; published online 30 July 2014
\end{abstract}

\section{INTRODUCTION}

Adolescence is a critical period for the initiation and escalation of alcohol use, and the development of alcohol abuse and dependence (Johnston et al, 2010). However, even within the framework of normative adolescent behavior there is considerable individual variation in drinking, which is likely to be related to variations in risk for later alcohol use disorder (AUD; Zucker, 2006) and to have genetic underpinnings.

Associations have been found between GABRA2 and adult alcohol dependence (eg, Edenberg et al, 2004). Within the haplotype block showing these associations, there are two common yin-yang haplotypes that account for the majority of the diversity in Caucasians (Enoch, 2008). The less frequent haplotype has been associated with alcoholics

\footnotetext{
*Correspondence: Dr MM Heitzeg, Department of Psychiatry, University of Michigan, 4250 Plymouth Road, Ann Arbor, Ml 481092700, USA, Tel: +734 232 0267, Fax: +734 998 7992, E-mail: mheitzeg@umich.edu

Received 7 March 20 I4; revised 13 June 20 I4; accepted I6 June 20 I4; accepted article preview online 30 June 2014
}

without comorbid drug dependence (Covault et al, 2004) and those with a family history of alcoholism (Fehr et al, 2006). This haplotype is also associated with childhood and adolescent phenotypic precursors to alcohol dependence, including externalizing problems throughout adolescence (Dick et al, 2009) and impulsiveness (Villafuerte et al, 2012).

Developmental changes occur in the relative importance of genetic and environmental effects on substance use and problems, with the importance of genetic factors increasing across adolescence into young adulthood (Kendler et al, 2008; Rose et al, 2001). However, few studies have investigated how risk conferred by specific genetic polymorphisms unfolds across development. One exception is the report of a GABRA2 association with conduct disorder (CD) symptoms in childhood, whereas an association with alcohol dependence symptoms was not evident until the mid-20's (Dick et al, 2006). This suggests that genetic influences are expressed differently at different developmental stages. To our knowledge, no studies have explored this within an imaging genetics framework by investigating how genetic risk may influence neural pathways differently across development. 
We investigated the neural pathway through which GABRA2 genetic variation may influence risk for alcoholism from childhood to young adulthood. We focused on the mesolimbic dopamine system, which is centrally involved in the reinforcing properties of drugs of abuse (Robinson and Berridge, 2000), undergoes change during adolescence (Luciana et al, 2012; Spear, 2011), and has been implicated in vulnerability to addiction (McBride and Li, 1998; Volkow et al, 2002). The nucleus accumbens (NAcc) is of particular importance; it contributes to directing behavior by integrating affective, contextual, and goal-directed information from the limbic system and prefrontal cortex (Grace et al, 2007). Inhibitory regulation of NAcc dopaminergic function comes from GABAergic influences (Steffensen et al, 1998). The GABRA2 gene is involved in encoding the $\alpha 2$ subunit of the $\gamma$-aminobutyric acid A receptor $\left(\mathrm{GABA}_{\mathrm{A}}\right)$, which is among the predominant receptor subtypes expressed in the NAcc (Schwarzer et al, 2001). GABRA2 gene deletion in a mouse model was found to reduce $\mathrm{GABA}_{\mathrm{A}}$-mediated electrophysiological responses in the NAcc (Dixon et al, 2010). Therefore, although the specific functional effects of GABRA2 variation are not understood (Tian et al, 2005), one possible neural mechanism through which GABRA2 may affect vulnerability to AUD is via this incentivemotivation system.

We investigated the developmental trajectory of NAcc function from childhood to young adulthood, the impact of GABRA2 on NAcc functioning, and whether GABRA2 effects change over the course of development. A monetary incentive delay (MID) task was used to probe NAcc functioning during the anticipation of monetary rewards in a high-risk sample. We focused on incentive anticipation owing to the evidence that NAcc dopamine functioning is specifically related to anticipatory, appetitive, or preparatory aspects of motivated behavior rather than consummatory aspects (Salamone and Correa, 2012). Functional MRI during the MID was collected at least once in 175 participants, with the majority $(n=151)$ undergoing repeated scanning at 1- to 2-year intervals. The present report is based on 473 scans covering ages 8-27 years. We investigated SNP rs279858; the minor (G) allele, a tag allele for the less frequent GABRA2 haplotype, has been associated with adult alcohol dependence (Covault et al, 2004) as well as adolescent externalizing problems (Dick et al, 2009) and impulsiveness (Villafuerte et al, 2012). Based on evidence of a positive relationship between NAcc activation to anticipation of reward during the MID and externalizing problems (Yau et al, 2012), impulsivity (Hahn et al, 2009) and sensation-seeking (Bjork et al, 2008a), we hypothesized that $G$ allele carriers would have heightened NAcc response. Furthermore, because adolescence is a time of increased reward-seeking and risk-taking, and heightened activity in the incentive-motivation system is proposed to be an important contributing factor to this (Casey and Jones, 2010; Galvan, 2010; Luciana et al, 2012), we expected that this effect would be most prominent during adolescence. Finally, we hypothesized that heightened NAcc response would mediate a relationship between genotype and problem drinking.

\section{MATERIALS AND METHODS}

\section{Participants}

At study onset, participants were 76 children/early adolescents aged 8-13 years and 99 late adolescents/young adults aged $18-23$ years. The majority $(n=151)$ underwent more than one scan (two to four scans; Table 1). All participants were drawn from the Michigan Longitudinal Study, an ongoing study of families with high levels of parental AUD along with a contrast sample of families without parental AUD (details in Supplementary Materials). Seventy-nine percent of participants $(n=139)$ had at least one parent with an AUD diagnosis $(\mathrm{FH}+)$.

Exclusionary criteria included: left-handed or ambidextrous; treatment with centrally active medications within past 6 months; history of psychosis or schizophrenia in first-degree relatives. The presence of most Axis I psychiatric or developmental disorders was exclusionary with the exception of $\mathrm{CD}$, attention deficit/hyperactivity disorder, or substance use disorder (SUD); CD and attention deficit/ hyperactivity disorder lie on a developmental spectrum with AUD/SUD and therefore elimination of these participants would eliminate part of the phenomenon of interest. Axis I disorders were assessed by a clinical psychologist based on DSM-IV criteria with the Diagnostic Interview ScheduleVersion 4 (Robins et al, 2000) at ages $18+$ years and the Diagnostic Interview Schedule-Child-Version 4 (Shaffer et al, 2000) at younger ages.

All participants were told to abstain from alcohol and illicit substances/recreational drugs for $48 \mathrm{~h}$ before scanning. In participants aged 15 years and older, urine drug screens were conducted immediately before the scan; positive results were exclusionary. In participants aged 14 years and younger, we relied on verbal confirmation of drug and alcohol abstinence on the day of the scan. Pregnancy

Table I Ages and Number of Scans by Age Group

Child/early adolescent

\begin{tabular}{|c|c|c|c|c|c|c|c|}
\hline & \multicolumn{3}{|c|}{ Age (years) } & \multicolumn{3}{|c|}{ Age (years) } & \multirow{2}{*}{$\begin{array}{c}\text { Total } \\
\text { N }\end{array}$} \\
\hline & $N$ & Range & Mean (SD) & $N$ & Range & Mean (SD) & \\
\hline Scan 2 (T2) & 59 & $|0.5-| 5 . \mid$ & $12.7(1.0)$ & 92 & $20.0-25.0$ & $22.5(1.4)$ & $|5|$ \\
\hline Scan 3 (T3) & 33 & $12.5-16.7$ & | $4.7(1.1)$ & 73 & $21.1-26.9$ & $24.2(1.4)$ & 106 \\
\hline
\end{tabular}

Late adolescent/young adult

Neuropsychopharmacology 
was exclusionary: all female participants were asked if they might be pregnant as part of the standard screening process for MRI. Females aged 15 years and older underwent a urine pregnancy test immediately before the scan; for younger females, we relied on verbal report.

After description of the study to the subjects, written informed consent, approved by the University of Michigan Medical School Institutional Review Board, was obtained. For those under age 18 years, written assent was obtained from the participant and written informed consent was obtained from at least one parent.

\section{Genotyping}

We selected SNP rs279858 (exon 5, K132K) from 11 SNPs in linkage disequilibrium that capture the information of a long haplotype block of $109 \mathrm{~kb}$, where previous associations have been reported with alcohol dependence and related traits. For SNP rs279858, TaqMan genotyping was performed using inventoried assays of primers and probes (Applied Biosystems, ABI, Foster City, CA). Twelve duplicates were included and no discrepancies were observed. See Supplementary Material for further details on genotyping methods. Frequency of the minor $(G)$ allele in this sample was $42 \%$; this did not differ significantly across the age groups $\left(\chi^{2}=2.8, p=0.235\right)$.

The samples were also genotyped for 150 ancestry informative markers (Hodgkinson et al, 2008), from which individual ethnic factor scores were computed (Supplementary Materials). The four ethnic factor scores explaining the highest variance (Table 2 ) were included as covariates in all genetic analyses to control population stratification.

\section{Substance Use}

Between ages 6 and 10 years, alcohol and drug use was assessed at 3-year intervals. Beginning at age 11 years, the Drinking and Drug History Form (Zucker et al, 1990) was filled out by participants annually. Details of substance use assessment are given in Supplementary Material. Annual drinking data (amount and frequency) are summed across all time points to calculate cumulative lifetime drinking volume (CDV). Participants are also asked about consequences and problems related to alcohol use (see Supplementary Table S1). The number of different drinking-related problems ever reported by the subject is cumulated by age for an annual cumulated alcohol problem measure.

\section{Functional MRI}

fMRI paradigm. Brain response during anticipation of incentive stimuli was probed using a modified MID task, as described previously (Yau et al, 2012; Supplementary Materials).

fMRI acquisition and data analysis. Whole-brain blood oxygen level-dependent (BOLD) functional images were acquired on a 3.0 Tesla GE Signa scanner (Milwaukee, WI) using a T2*-weighted single-shot combined spiral in/out sequence with the following imaging parameters: repetition time $=2000 \mathrm{~ms}$, echo time $=30 \mathrm{~ms}$, flip angle $=90^{\circ}$; field-ofview $=200 \mathrm{~mm}$; matrix size $=64 \times 64$; in plane resolution $=$
$3.12 \times 3.12 \mathrm{~mm}^{2}$; and slice thickness $=4 \mathrm{~mm}$. A high-resolution anatomical T1 scan was obtained for spatial normalization (three-dimensional spoiled gradient recalled echo, repetition time $=25 \mathrm{~ms}$, min echo time, field-of-view $=25 \mathrm{~cm}$, $256 \times 256$ matrix, slice thickness $=1.4 \mathrm{~mm}$ ).

Functional images were reconstructed using an iterative algorithm (Fessler et al, 2005). Head motion was corrected using the FSL 5.0.2.2 analysis tools library (Analysis Group, FMRIB, Oxford, UK), slice-acquisition timing was corrected using SPM8 (Wellcome Institute of Cognitive Neurology, London, UK). Runs exceeding $3 \mathrm{~mm}$ translation or $3^{\circ}$ rotation in any direction were excluded (younger groupbaseline: $8.0 \%$; T2: 1.9\%; T3\&T4: $0 \%$; older groupbaseline: $1.4 \%$; T2: 1.5\%; T3: 0.7\%; T4: 1.8\%). Remaining image processing was completed using SPM8 (Wellcome Institute of Cognitive Neurology, London, UK). Functional images were spatially normalized to a standard stereotactic space as defined by the Montreal Neurological Institute. A 6-mm full-width half-maximum Gaussian spatial smoothing kernel was applied to improve signal-to-noise ratio and account for individual differences in anatomy.

Individual analysis was completed using a general linear model. Regressors of interest for all events (cues: win $\$ 0.20$, win $\$ 5.0$, lose $\$ 0.20$, lose $\$ 5.0$ and neutral $\$ 0$; anticipation delay for each cue type; positive outcome for each cue type; negative outcome for each cue type) were included and convolved with the canonical hemodynamic response function. Motion parameters and white matter signal intensity were modeled as nuisance regressors to remove residual motion artifacts and capture non-task-related noise, respectively. Scanner drift and other low-frequency noise were removed from the image time series using a 128-s high-pass filter.

Owing to our interest in incentive anticipation, the present report focuses on the contrast between anticipation during monetary gain trials $v s$ neutral trials (we refer to this contrast in the remaining text as 'reward anticipation'). To avoid circularity of statistical inference, which can occur by defining volumes of interest (VOI) based on observed contrast activation, and given an a priori interest in the NAcc, anatomical masks of NAcc were created as described previously (Bjork et al, 2008b; Yau et al, 2012; Supplementary Materials) and analyses were restricted to this VOI. Left and right NAcc VOI effect sizes were calculated from parameter estimates using MarsBaR region of interest toolbox (Brett et al, 2002) for each incentive amount ( $\$ 0.20$ and $\$ 5$ ) compared with neutral.

Effects of hemisphere and incentive amount were investigated using repeated-measures ANOVA (Supplementary Materials), revealing no main effects of, or interactions with hemisphere; therefore, NAcc VOI effect sizes for $\$ 0.20$ and $\$ 5$ were linearly combined and averaged across hemispheres.

Intraclass correlation coefficient was calculated to test reliability of NAcc activation across sessions and was found to be acceptable (intraclass correlation coefficient $=0.597$; see Supplementary Materials).

\section{Statistical Analysis}

Linear mixed-effects models (LMMs). To determine the pattern of NAcc activation across the age-range, a LMM was run in SPSS. LMMs account for correlation between repeated measurements within subjects while also allowing 
Table 2 Subject Characteristics by Genotype

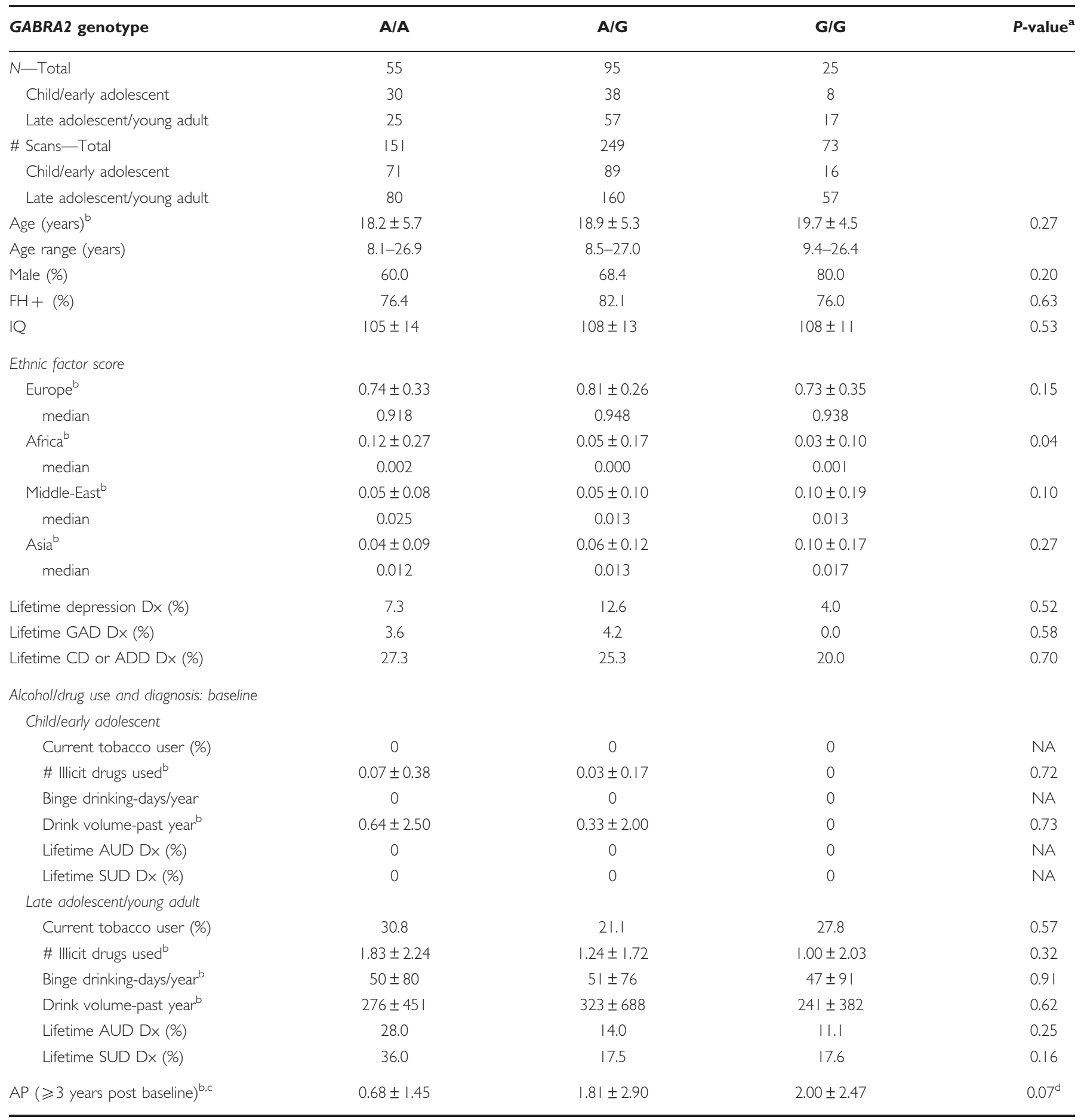

Abbreviations: ADD, attention deficit disorder; AP, alcohol problems; AUD, alcohol use disorder; CD, conduct disorder; Dx, diagnosis; FH+, one or both parents have a lifetime diagnosis of alcohol abuse or dependence; GAD, generalized anxiety disorder; NA, not applicable due to zero values; SUD, substance use disorder. ${ }^{a} P$-values represent the results of Pearson $\chi^{2}$ or ANOVA tests.

bData presented as mean \pm standard deviation.

'The number of alcohol problems reported over at least 3 years following baseline (range: 3-6 years; mean: 4 years). These data were available in $n=104$ (AA $n=30$; $A G n=56$; $G G n=18$ ).

${ }^{d} p=0.04$ when linear relationship is tested using Pearson correlation with \# of minor (G) alleles.

for an unequal number of repeated measurements across subjects (Gueorguieva and Krystal, 2004). The dependent variable was NAcc activation during reward anticipation, as defined above. Time point (baseline, T2, T3, T4) was included as a repeated effect, subject as a random factor, and age-group, linear age, and quadratic age were included 
simultaneously as fixed-effect covariates. Additional factors that may impact the developmental trajectory of NAcc activation were also explored (gender, FH, CDV). Schwarz's Bayesian information criteria (Schwarz, 1978) were used to find best-fitting variance-covariance structure; determined to be scaled-identity.

To test the impact of GABRA2 on the developmental trajectory of NAcc activation, genotype was added as a fixed factor; main effects and genotype by age interactions were investigated.

Repeated-measures analysis. To confirm findings from the LMMs, follow-up analyses were conducted in a subset from each age-group to investigate within-subject effects. In the younger group, individuals had to have a baseline scan conducted before age 13 years and a follow-up scan at 13 years or older $(n=43)$. In the older group, individuals had to have a baseline scan conducted before age 21 years and a follow-up scan at 21 years or older $(n=58)$. Repeatedmeasure ANCOVAs, with time (baseline, follow-up) as a within-subject factor were used to determine within-subject developmental effects for each group. To test the impact of GABRA2, genotype was added as a between-subject factor.

Relationship with alcohol problems. To investigate the hypothesis that GABRA2 genetic variation is related to problem drinking through its effects on NAcc responding to incentive stimuli, a bias-corrected bootstrapped indirect effect analysis was conducted using an SPSS macro (Preacher et al, 2007). GABRA2 genotype was the independent variable, NAcc activation at baseline was the mediator, and the number of alcohol problems reported over at least 3 years following baseline (AP; range: 3-6 years; mean: 4 years) was the dependent variable. AP was available for 104 participants (see Table 2). Gender, FH, and CDV at baseline were included as covariates. Bootstrapping (10 000 resamples) was performed to determine bias-corrected $95 \%$ confidence intervals.

For all genetic analyses, genotype was coded as number of minor alleles $(\mathrm{AA}=0, \mathrm{AG}=1, \mathrm{GG}=2)$, which makes the fewest assumptions and is consistent with literature reporting a dose-dependent allelic effect (Covault et al, 2004; Fehr et al, 2006), and ethnic factor scores were included as covariates. Drinking variables (CDV and AP) were squareroot transformed because of non-normal distribution of raw values.

Supplementary analyses. All analyses were conducted in Caucasians only and no major divergences from the main findings were noted; although $p$-values were reduced, effects trended in the same direction. Analyses were also conducted excluding participants with $\mathrm{CD}$ or ADD diagnoses; results indicate these participants were not driving the findings. Further analyses were conducted to explore differences based on FH and gender. Finally, supplementary analyses investigated age-group by linear age interactions to verify that the developmental trajectory identified was not an artifact of investigating age effects across two non-overlapping age-groups. All supplementary analyses are reported completely in Supplementary Material.

\section{RESULTS}

\section{Subject Characteristics and Task Performance}

There were no genotype differences in gender, age, $\mathrm{FH}$, substance use, or diagnosis of psychopathology (Table 2). In the older group, there was no significant effect of genotype on lifetime diagnosis of AUD/SUD.

Effect of incentive amount on performance is reported in Supplementary Materials. LMMs including age, age ${ }^{2}$, genotype, age $\times$ genotype, and age ${ }^{2} \times$ genotype revealed no main effects of, or interactions with, genotype on accuracy to combined reward ( $\$ 0.20$ and $\$ 5)$ compared with neutral trials ( $p$ 's $>0.14)$ as expected, given the task is individually calibrated to target $60 \%$ accuracy. There were also no genotype effects on accuracy to reward or neutral trials when investigated separately $(p$ 's $>0.40)$.

There were no main effects or interactions of genotype on reaction time during reward trials compared with neutral trials $(p$ 's $>0.50)$. However, when reaction time was investigated for each incentive condition separately, there was a significant effect of genotype during reward trials $\left(\mathrm{F}_{272,2}=4.8, \quad p=0.009\right)$ but not during neutral trials $\left(\mathrm{F}_{289,2}=2.4, p=0.092\right) ; \mathrm{G}$ homozygotes responded faster than A allele carriers during reward trials, with a trend for faster responding during neutral trials.

\section{Developmental Effect: LMMs}

Both age groups activated the NAcc during reward anticipation (Supplementary Materials). The LMM revealed a significant quadratic effect of age on NAcc activation during reward anticipation $\left(\mathrm{F}_{453,1}=9.1, p=0.003\right)$ across all 473 scans. Age-group and linear age terms were not significant $(p>0.4)$. Activation of the NAcc to reward anticipation from childhood to early adulthood followed an inverted U-shaped pattern (Figure 1a). This was further supported by exploratory linear regression showing age ${ }^{2}$ significantly improved model fit above age-group and linear age (Supplementary Materials). When gender and FH were included, the quadratic age term remained significant (age ${ }^{2}$ : $\left.\mathrm{F}_{452,1}=9.4, p=0.002\right)$; there were no other significant variables $(p$ 's $>0.4)$. Likewise, the addition of CDV as a covariate did not significantly impact the results (age ${ }^{2}$ : $\mathrm{F}_{456,1}=9.7, p=0.002$; all other $p$ 's $>0.4$ ).

\section{Developmental Effect: Within Subjects}

Repeated-measures ANCOVAs found effects of time: in the younger group, activation of the NAcc increased from childhood to adolescence in the same subjects $\left(\mathrm{F}_{42,1}=7.0\right.$, $p=0.011$; Figure 2a); in the older group, activation of the NAcc decreased from later adolescence to young adulthood in the same subjects $\left(\mathrm{F}_{57,1}=8.4, p=0.005\right.$; Figure $\left.1 \mathrm{~b}\right)$.

\section{Interaction between Development and GABRA2: LMMs}

There was a main effect of rs 279858 on NAcc activation during reward anticipation (GG $>A G \& A A ; F_{266,2}=5.1$, $p=0.007)$, a main effect of age ${ }^{2}\left(F_{460,1}=10.8, p=0.001\right)$ and an age ${ }^{2}$ by genotype interaction $\left(\mathrm{F}_{454,2}=3.0, p=0.050\right.$; Figure 2a) across all 473 scans. Age-group and linear age were not significant $(p>0.6)$. When gender and FH were 

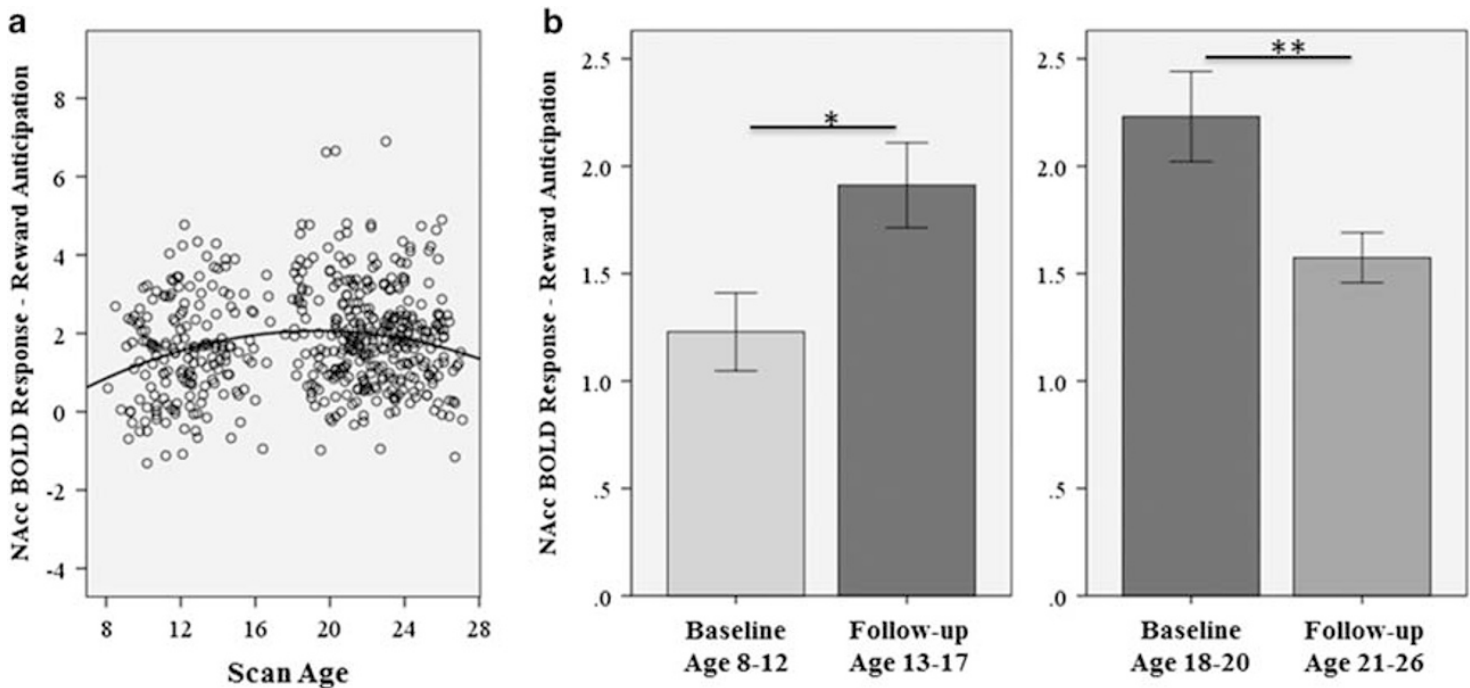

Figure I Illustration of significant quadratic age effect on Nacc BOLD activation to reward anticipation. (a) Across all scans covering ages 8-27 years. Note: this figure includes repeated-measures within individuals as described in Table I (473 measures on I75 individuals). (b) Within-subject time effect on NAcc BOLD activation to reward anticipation in the child/young adolescent group (left), with baseline scan in childhood and a follow-up scan in early adolescence $(n=43)$; and the late adolescent/young adult group (right), with baseline scan in late adolescence and follow-up scan in young adulthood $(n=58)$. Error bars represent I standard error, $* p<0.05$, *** $p<0.01$.
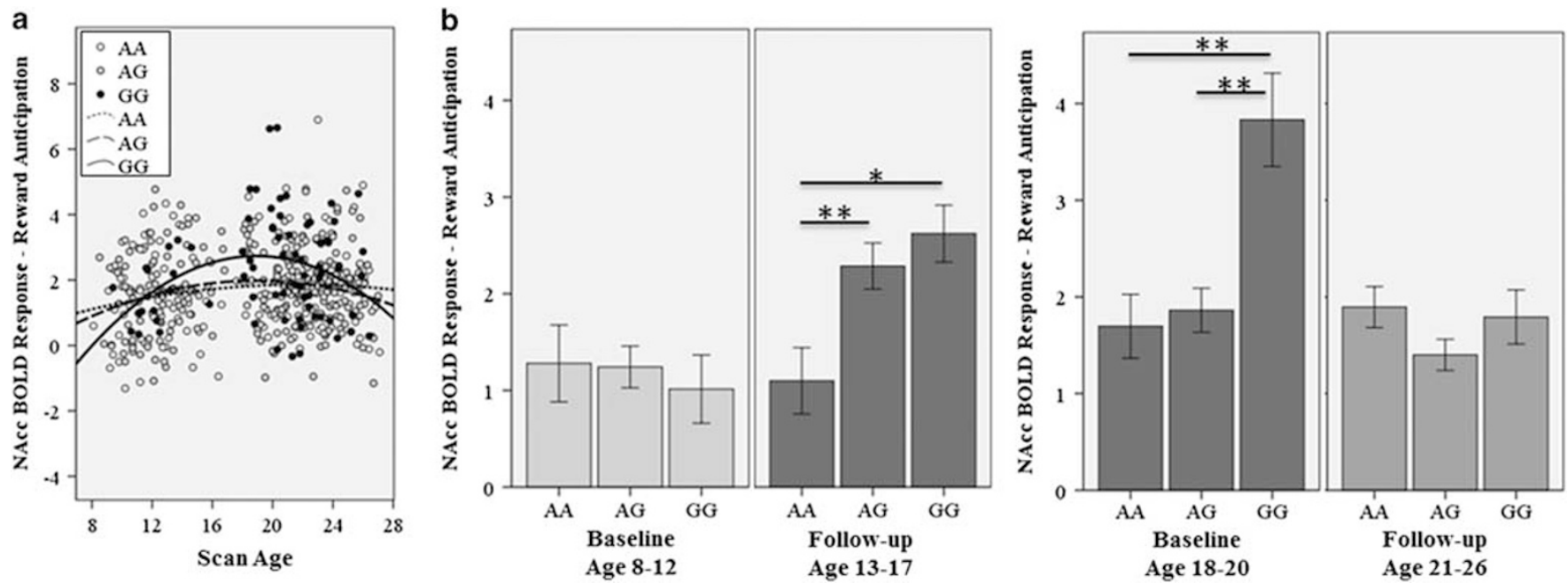

Figure 2 Age by genotype interaction on NAcc BOLD activation to reward anticipation. (a) Age-squared by genotype interaction across the ages $8-27$ years. Note: this figure includes repeated measures within individuals as described in Table I (473 measures on 175 individuals). (b) Within-subject time by genotype interaction in the child/early adolescent group (left), with baseline scan in childhood and a follow-up scan in adolescence ( $n=43$; AA $n=15$; AG $n=23 ; G G n=5$ ); and the late adolescent/young adult group (right), with baseline scan in late adolescence and follow-up scan in young adulthood ( $n=58$; $A A n=14 ; A G n=31$; $G G n=13$ ). Error bars represent I standard error, $* p<0.05$, $* * x<0.01$.

included as covariates, the main effects remained significant (genotype: $\quad \mathrm{F}_{265,2}=5.2, \quad p=0.006 ; \quad$ age $^{2}: \quad \mathrm{F}_{458,1}=10.8$, $p=0.001)$; genotype $\times$ age $^{2}$ was reduced to trend-level significance $\left(\mathrm{F}_{452,2}=2.7, p=0.056\right)$. There were no other significant variables in the model $(p$ 's $>0.4)$. The inclusion of $\mathrm{CDV}$ as a covariate did not impact the main effects (genotype: $\quad \mathrm{F}_{264,2}=5.5, \quad p=0.005 ; \quad$ age $^{2}: \quad \mathrm{F}_{457,1}=11.5$, $p=0.001$ ) but further reduced the significance-level of the genotype $\times$ age $^{2}$ interaction $\left(\mathrm{F}_{451,2}=2.5, p=0.087\right)$. Post-hoc analyses, including all covariates, investigated age effects for each genotype separately; there were significant quadratic effects of age in $\mathrm{G}$ allele carriers $\left(\mathrm{GG}: \mathrm{F}_{61,1}=5.5\right.$, $\left.p=0.022 ; \mathrm{AG}: \mathrm{F}_{233,1}=9.8, p=0.002\right)$ but not A homozygotes $\left(\mathrm{F}_{140,1}=0.1, p=0.908\right)$.

\section{Interaction between Development and GABRA2: Within Subjects}

Repeated-measures ANCOVA in the younger group $(n=43)$ including all covariates found no main effects of time $\left(\mathrm{F}_{33,1}=\right.$ $1.7, p=0.207)$ or genotype $\left(\mathrm{F}_{33,2}=2.8, p=0.077\right)$ but a genotype by time interaction approached significance $\left(\mathrm{F}_{33,2}=3.1\right.$, $p=0.057)$. Post-hoc ANCOVAs investigating genetic effects for 
a
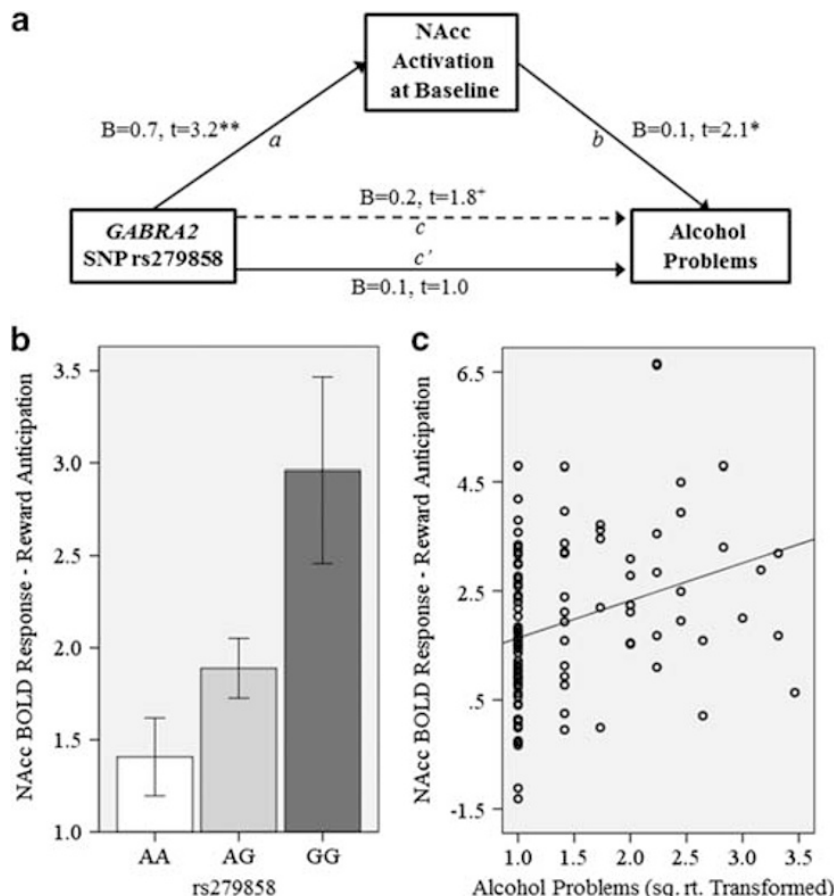

Figure 3 (a) Indirect effect model. Number of minor (G) alleles is independent variable. Nucleus accumbens (NAcc) activation at baseline scan is the mediator. Number of alcohol problems reported over 3-6 years following baseline (average $=4$ years) is the dependent variable. The model included the following covariates: gender, family history of alcoholism, four ethnic factors, and cumulative lifetime drinking volume at time of baseline scan. Unstandardized coefficients (b) are given. (b) Illustration of a path effect demonstrating increasing NAcc activation with increasing number of $G$ alleles. (c) Illustration of $b$ path with increased NAcc activation at baseline associated with increased number of alcohol problems in the following 3-6 years. ${ }^{+} p<0.10, * 0<0.05, * * * 60.001$.

each time point separately found no effect during childhood (baseline: $\mathrm{F}_{33,2}=0.0, p=0.966$ ), but a significant genotype effect during adolescence (follow-up: GG\&AG $>A A ; F_{33,2}=$ 5.4, $p=0.009$ ) in the same subjects (Figure $2 b$ ).

In the older group $(n=58)$, there was a main effect of genotype $\left(\mathrm{F}_{48,2}=10.0, p<0.001\right)$ and a genotype by time interaction $\left(\mathrm{F}_{48,2}=6.1, p=0.004\right)$. There was no main effect of time $\left(\mathrm{F}_{48,1}=0.8, p=0.366\right)$. Post-hoc ANCOVAs found a significant effect of genotype during later adolescence (baseline: GG $>$ AG\&AA; $\mathrm{F}_{48,2}=10.8, p<0.001$ ), but not young adulthood (follow-up: $\mathrm{F}_{48,2}=1.0, p=0.372$ ) in the same subjects (Figure $2 \mathrm{~b}$ ).

\section{Indirect Effect of GABRA2 on Alcohol Problems via NAcc}

Bivariate correlations among the main variables included in the model were significant (rs279858 and NAcc: $r=0.33$, $p<0.001$; NAcc and AP: $r=0.27, p=0.006$; rs279858 and AP: $r=0.20, p=0.047)$. Figure 3 illustrates the results of the indirect model. There was a significant relationship between rs279858 and NAcc activation (a path; illustrated in Figure $3 \mathrm{~b}$ ), and a positive relationship between NAcc activation and later alcohol problems ( $b$ path; illustrated in Figure 3c). The total model was significant $\left(R^{2}=0.15, \mathrm{~F}=2.3\right.$, $p=0.031)$. Bootstrap $(n=10000)$ bias-corrected and accelerated $95 \%$ confidence intervals (lower limit $=0.007$, upper limit $=0.178$ ) indicated a significant indirect effect of rs279858 on later alcohol problems via NAcc activation during reward anticipation. Confidence intervals remained significant when age at baseline was added to the model as a covariate $($ lower limit $=0.002$, upper limit $=0.167$ ).

\section{DISCUSSION}

The goal of this work was to investigate a neural mechanism through which GABRA2 genetic variation may influence risk for alcoholism and the possible developmental variability in its operation. We found that GABRA2 genotype is associated with individual differences in NAcc activation during incentive anticipation specifically during adolescence. This was demonstrated across the entire age range as a genotype by age-squared interaction, and also within-subjects as a genotype by time interaction, with an effect of genotype only during adolescence in both age groups. Furthermore, NAcc activation was found to mediate the relationship between genotype and later alcohol problems. These associations are not driven by subjects with ADD or CD diagnoses, as the results are similar when these individuals are excluded.

The $\mathrm{G}$ allele of GABRA2 SNP rs279858 has been associated with adult alcohol dependence (Covault et al, 2004), elevated externalizing problems throughout adolescence (Dick et al, 2009), impulsiveness (Villafuerte et al, 2012), and increased insula activation during incentive anticipation (Villafuerte et al, 2012). We show that activation of the NAcc to incentive stimuli is heightened in G carriers during adolescence. Previous studies have reported relationships between increased NAcc activation during reward anticipation and behavioral traits related to vulnerability to addictive disorders, such as impulsiveness (Forbes et al, 2009; Hahn et al, 2009), sensation-seeking (Bjork et al, 2008a), and externalizing behaviors (Yau et al, 2012). Consistent with this, we observed a relationship between heightened activation of the NAcc and later alcohol problems. Furthermore, the present study provides an additional link, demonstrating a genetic influence on these relationships. Specifically, GABRA2 genotype had an indirect effect on alcohol problems via hyperactivation of the NAcc, extending the current knowledge regarding the neural pathway through which GABRA2 may affect risk for alcoholism.

$G A B R A 2$ gene deletion in mice has been shown to reduce electrophysiological responses mediated by $\mathrm{GABA}_{\mathrm{A}}$ receptors in the NAcc, abolish behavioral sensitization to cocaine and cocaine's ability to facilitate lever-pressing for a conditioned reinforcer (Dixon et al, 2010), and abolish cue-associated responding when no cocaine was available (Dixon et al, 2014). This supports a role of GABRA2 genetic variation in individual differences in incentive motivation, with the NAcc as a critical neural mediator of this effect. Importantly, GABRA2 gene deletion did not impact alcohol self-administration (Dixon et al, 2012), cocaine self-administration, or reinstatement of cocaine-seeking after extinction (Dixon et al, 2014), suggesting that the mechanism of this effect does not involve the signaling of drug reward. Rather, Dixon and colleagues conclude that manipulation of the $\mathrm{GABA}_{\mathrm{A}} \propto 2$ subunit has the most consistent impact on the expression of cue-associated behavior-or the 
'energizing' aspects of reward-seeking (Dixon et al, 2014). The present work is consistent with this, showing that GABRA2 genetic variation is associated with individual differences in NAcc response during the anticipatory phase of the MID, when participants are preparing to respond for potential reward; specifically, G homozygotes showed heightened NAcc activation compared with A allele carriers during reward anticipation and also had faster responses to reward trials. This is also consistent with evidence that NAcc dopamine is involved in anticipatory, appetitive, or preparatory aspects of motivation, including behavioral activation, approach behavior, and sustained task engagement, as opposed to consummatory aspects (Salamone and Correa, 2012). Therefore, a possible mechanism through which heightened NAcc response to incentives may confer risk for problem drinking is via enhancement of behavioral activation aspects of motivation toward alcohol cues.

An important aspect of this work is the demonstration of developmental effects in NAcc anticipatory response. An inverted U-shaped curve was found to describe the developmental trajectory of NAcc response across the entire age range. This pattern held when lifetime drinking volume was added to the model as a covariate and therefore did not appear to be driven by the amount of substance use in the older group. Furthermore, the pattern was supported within-subjects in each age group. Adolescence is a time of increased reward-seeking and risk-taking, with heightened activity in the incentive-motivation system proposed to be an important contributing factor to this (Casey and Jones, 2010; Galvan, 2010; Luciana et al, 2012). Crosssectional studies using fMRI in conjunction with a variety of reward paradigms have provided support for this view (Ernst et al, 2005; Galvan et al, 2006; Van Leijenhorst et al, 2010). However, others have reported hyporeactivity to reward in adolescents (Bjork et al, 2004; Bjork et al, 2010; Geier et al, 2010). A number of excellent reviews have described possible explanations for these discrepancies, including differences in task design, phases of reward processing, and definitions of adolescence (Galvan, 2010; Spear, 2011). Therefore, we limit this discussion to studies that investigated the anticipation phase during an MID task. In a group comparison between adolescents aged 12-17 years $(n=24)$ and adults aged 22-42 years $(n=24)$, reduced activation of the NAcc during anticipation of reward was found in adolescents (Bjork et al, 2010). However, a comparison of 10 - to 17 -year olds $(n=24)$ with 22 - to 48 year olds $(n=30)$ found reduced activation in the putamen, but not the ventral striatum (Cho et al, 2012). Differences across these reports and the present work may be due in part to definition of age groups, which could introduce confounds (Galvan, 2010; Luna et al, 2010). Importantly, the use of distinct age groups separated by 4 years does not allow for a quadratic function of age to be modeled. Indeed, when restricting our data to a group comparison between ages 12-17 and ages 22-27 years in an exploratory analysis, we observed a trend for less activation in the 12- to 17-yearold group ( $p=0.12$; data not shown). In contrast, a study of 10 - 25-year olds $(n=39)$ modeled linear and quadratic age effects across subjects as opposed to using age groups. They found a positive linear relationship between age and ventral striatal activation to anticipation of reward and, in contrast to the present report, the quadratic term did not signifi- cantly contribute beyond the linear term (Hoogendam et al, 2013). It is possible that the more thorough interrogation of the developmental continuum reported on here-with a broader age range $(8-27)$, larger sample $(n=175)$, and repeated measures (473 sessions) - is necessary to reveal the inverted U-shaped trajectory of activation to reward anticipation during the MID. Alternatively, given that this was a primarily high-risk sample, the observed trajectory may specifically reflect developmental patterns in those with heightened risk conferred by environmental and genetic influences attributable to a family history of alcoholism. Indeed, supplementary analyses found a significant effect of quadratic age in participants with a family history of alcoholism (79\% of sample) but not in those without (presented in Supplementary Material); however, this may be due to the smaller sample size in the latter group. Largescale imaging studies including repeated-measures in representative samples are required to determine if normative development follows this same trajectory.

Most importantly, age was found to interact with GABRA2 genotype, resulting in different trajectories of NAcc activation to incentive stimuli; $G$ allele carriers showed an inverted U-shaped developmental curve, whereas AA homozygotes showed no evidence of age-related changes. This is a critical finding; it demonstrates a specific genetic influence on adolescent development as it relates to individual differences in the evolution of risky behavior. Despite the vast literature characterizing adolescence as a period of heightened reward-seeking and risk-taking, not all teens demonstrate these behavioral tendencies and it is important to understand not only group-based profiles, but also individual differences in behavior. The present work suggests that genetic variation in GABRA2 affects individual differences in adolescent risk-taking via effects on the dopaminergic inventive-motivation system. However, to date, the specific functional effects of GABRA2 allelic variation have not been identified. Further research is necessary to understand the mechanism through which GABRA2 variation impacts the functioning of this system throughout development.

Some potential caveats about this work should be noted. First, as noted above, this was a primarily high-risk sample, limiting the generalizability of the findings. Second, although supplementary analyses did not reveal any significant effects of gender, findings were more robust in males than females. A larger sample of female participants is necessary to determine whether there are gender differences in GABRA2 effects on NAcc responding to incentive stimuli across development. Data collection in this sample is ongoing and this will be an important focus of future work. Third, it should be noted that there is a large amount of variation in BOLD activation even within genotype groups. There are numerous potential sources of variation not accounted for in the current study, including environmental influences such as history of stressful events and past experiences with reward, which can impact functioning of the mesolimbic dopamine system (Pitchers et al, 2010). Furthermore, constraining influences from prefrontal regions likely introduce an additional source of variation in the development and functioning of this system. Future work investigating the impact of GABRA2 genetic variation on the interaction between NAcc and prefrontal cortex 
functioning from childhood to adulthood is necessary for a fuller understanding of these relationships. Finally, the developmental trajectory described here was extrapolated from longitudinal data collected in two groups with no age overlap. Although the supplementary analyses investigating age-group by linear age interactions suggest that the developmental trajectory identified was not an artifact of investigating age effects across two non-overlapping age groups, future work is necessary to confirm these findings using longitudinal measures collected from childhood to young adulthood within the same subjects.

We found that GABRA2 affects individual differences in incentive responding during a critical developmental period, leading indirectly to problem drinking. These findings represent an important step toward understanding individual differences in how risk for substance abuse unfolds across development. Understanding individual differences in risk mechanisms may allow a better understanding of differential responsiveness to preventative interventions, leading to more individualized targeting. Furthermore, timing is central to prevention in terms of choosing when to begin intervention, and also determining the specific mechanisms to target. The present work suggests that incentive motivation is a promising target specifically during adolescence for some individuals. Further research is indicated to investigate whether heightened incentive sensitivity is a malleable target for intervention during the adolescent period.

\section{FUNDING AND DISCLOSURE}

The authors declare no conflict of interest.

\section{ACKNOWLEDGEMENTS}

This work was supported by R01 DA027261 to MMH, JKZ, and RAZ, R01 AA12217 to RAZ and MMH, R37 AA07065 to RAZ, and K01 DA020088 to MMH, and by the Phil F. Jenkins Research Fund (JKZ). This research was also supported in part by the Intramural Research Program of the National Institute on Alcohol Abuse and Alcoholism, NIH (MAE).

\section{REFERENCES}

Bjork JM, Knutson B, Fong GW, Caggiano DM, Bennett SM, Hommer DW (2004). Incentive-elicited brain activation in adolescents: similarities and differences from young adults. J Neurosci 24: 1793-1802.

Bjork JM, Knutson B, Hommer DW (2008a). Incentive-elicited striatal activation in adolescent children of alcoholics. Addiction 103: 1308-1319.

Bjork JM, Smith AR, Chen G, Hommer DW (2010). Adolescents, adults and rewards: comparing motivational neurocircuitry recruitment using fMRI. PLoS One 5: e11440.

Bjork JM, Smith AR, Hommer DW (2008b). Striatal sensitivity to reward deliveries and omissions in substance dependent patients. Neuroimage 42: 1609-1621.

Brett MAnton J-LValabregue RPoline JB (eds) (2002). Region of interest analysis using an SPM toolbox. 8th International Conference on Functional Mapping of the Human Brain. June 2-6 Sendai, Japan.
Casey BJ, Jones RM (2010). Neurobiology of the adolescent brain and behavior: implications for substance use disorders. $\mathrm{J} \mathrm{Am}$ Acad Child Adolesc Psychiatry 49: 1189-1201 quiz 1285.

Cho YT, Fromm S, Guyer AE, Detloff A, Pine DS, Fudge JL et al (2012). Nucleus accumbens, thalamus and insula connectivity during incentive anticipation in typical adults and adolescents. Neuroimage 66C: 508-521.

Covault J, Gelernter J, Hesselbrock V, Nellissery M, Kranzler HR (2004). Allelic and haplotypic association of GABRA2 with alcohol dependence. Am J Med Genet B Neuropsychiatr Genet 129B: 104-109.

Dick DM, Bierut L, Hinrichs A, Fox L, Bucholz KK, Kramer J et al (2006). The role of GABRA2 in risk for conduct disorder and alcohol and drug dependence across developmental stages. Behav Genet 36: 577-590.

Dick DM, Latendresse SJ, Lansford JE, Budde JP, Goate A, Dodge KA et al (2009). Role of GABRA2 in trajectories of externalizing behavior across development and evidence of moderation by parental monitoring. Arch Gen Psychiatry 66: 649-657.

Dixon CI, Halbout B, King SL, Stephens DN (2014). Deletion of the GABA alpha2-subunit does not alter self administration of cocaine or reinstatement of cocaine seeking. Psychopharmacology (Berl) 231: 2695-2703.

Dixon CI, Morris HV, Breen G, Desrivieres S, Jugurnauth S, Steiner $\mathrm{RC}$ et al (2010). Cocaine effects on mouse incentive-learning and human addiction are linked to alpha2 subunit-containing GABAA receptors. Proc Natl Acad Sci USA 107: 2289-2294.

Dixon CI, Walker SE, King SL, Stephens DN (2012). Deletion of the gabra2 gene results in hypersensitivity to the acute effects of ethanol but does not alter ethanol self administration. PLoS One 7: e47135.

Edenberg HJ, Dick DM, Xuei X, Tian H, Almasy L, Bauer LO et al (2004). Variations in GABRA2, encoding the alpha 2 subunit of the GABA(A) receptor, are associated with alcohol dependence and with brain oscillations. Am J Hum Genet 74: 705-714.

Enoch MA (2008). The role of GABA(A) receptors in the development of alcoholism. Pharmacol Biochem Behav 90: 95-104.

Ernst M, Nelson EE, Jazbec S, McClure EB, Monk CS, Leibenluft E et al (2005). Amygdala and nucleus accumbens in responses to receipt and omission of gains in adults and adolescents. Neuroimage 25: 1279-1291.

Fehr C, Sander T, Tadic A, Lenzen KP, Anghelescu I, Klawe C et al (2006). Confirmation of association of the GABRA2 gene with alcohol dependence by subtype-specific analysis. Psychiatr Genet 16: 9-17.

Fessler J, Lee S, Olafsson V, Shi H, Noll D (2005). Toeplitz-based iterative image reconstruction for MRI with correction for magnetic field inhomogeneity. IEEE Trans Signal Proc 53: 3393-3402.

Forbes EE, Brown SM, Kimak M, Ferrell RE, Manuck SB, Hariri AR (2009). Genetic variation in components of dopamine neurotransmission impacts ventral striatal reactivity associated with impulsivity. Mol Psychiatry 14: 60-70.

Galvan A (2010). Adolescent development of the reward system. Front Hum Neurosci 4: 6.

Galvan A, Hare TA, Parra CE, Penn J, Voss H, Glover G et al (2006). Earlier development of the accumbens relative to orbitofrontal cortex might underlie risk-taking behavior in adolescents. J Neurosci 26: 6885-6892.

Geier CF, Terwilliger R, Teslovich T, Velanova K, Luna B (2010). Immaturities in reward processing and its influence on inhibitory control in adolescence. Cereb Cortex 20: 1613-1629.

Grace AA, Floresco SB, Goto Y, Lodge DJ (2007). Regulation of firing of dopaminergic neurons and control of goal-directed behaviors. Trends Neurosci 30: 220-227.

Gueorguieva R, Krystal JH (2004). Move over ANOVA: progress in analyzing repeated-measures data and its reflection in papers 
published in the Archives of General Psychiatry. Arch Gen Psychiatry 61: 310-317.

Hahn T, Dresler T, Ehlis AC, Plichta MM, Heinzel S, Polak T et al (2009). Neural response to reward anticipation is modulated by Gray's impulsivity. Neuroimage 46: 1148-1153.

Hodgkinson CA, Yuan Q, Xu K, Shen PH, Heinz E, Lobos EA et al (2008). Addictions biology: haplotype-based analysis for 130 candidate genes on a single array. Alcohol Alcohol 43: 505-515.

Hoogendam JM, Kahn RS, Hillegers MH, van Buuren M, Vink M (2013). Different developmental trajectories for anticipation and receipt of reward during adolescence. Dev Cogn Neurosci 6C: 113-124.

Johnston LD, O’Malley PM, Bachman JG, Schulenberg JE (2010). Monitoring the Future: National Survey Results on Drug Use, 1975-2009: Volume1, College Students and Adults Ages 19Secondary School Students. National Institute on Drug Abuse, US Government Printing Office: Bethesda MD.

Kendler KS, Schmitt E, Aggen SH, Prescott CA (2008). Genetic and environmental influences on alcohol, caffeine, cannabis, and nicotine use from early adolescence to middle adulthood. Arch Gen Psychiatry 65: 674-682.

Luciana M, Wahlstrom D, Porter JN, Collins PF (2012). Dopaminergic modulation of incentive motivation in adolescence: age-related changes in signaling, individual differences, and implications for the development of self-regulation. Dev Psychol 48: 844-861.

Luna B, Velanova K, Geier CF (2010). Methodological approaches in developmental neuroimaging studies. Hum Brain Mapp 31: 863-871.

McBride WJ, Li TK (1998). Animal models of alcoholism: neurobiology of high alcohol-drinking behavior in rodents. Crit Rev Neurobiol 12: 339-369.

Pitchers KK, Balfour ME, Lehman MN, Richtand NM, Yu L, Coolen LM (2010). Neuroplasticity in the mesolimbic system induced by natural reward and subsequent reward abstinence. Biol Psychiatry 67: 872-879.

Preacher KJ, Rucker DD, Hayes AF (2007). Addressing moderated mediation hypotheses: theory, methods, and prescriptions. Multivariate Behav Res 42: 185-227.

Robins L, Cottler LB, Bucholz KK, Compton WM, North CS, Rourke K (2000). Diagnostic Interview Schedule for the DSM-IV (DSM-IV). Washington University School of Medicine: St Louis, MO.

Robinson TE, Berridge KC (2000). The psychology and neurobiology of addiction: an incentive- sensitization view. Addiction 95(Suppl 2)): S91-S117.

Rose RJ, Dick DM, Viken Rj, Kaprio J (2001). Gene-environment interaction in patterns of adolescent drinking: regional residency moderates longitudinal influences on alcohol use. Alcohol Clin Exp Res 25: 637-643.
Salamone JD, Correa M (2012). The mysterious motivational functions of mesolimbic dopamine. Neuron 76: 470-485.

Schwarz G (1978). Estimating the dimensions of a model. Ann Stat 6: 241-263.

Schwarzer C, Berresheim U, Pirker S, Wieselthaler A, Fuchs K, Sieghart W et al (2001). Distribution of the major gammaaminobutyric acid(A) receptor subunits in the basal ganglia and associated limbic brain areas of the adult rat. J Comp Neurol 433: 526-549.

Shaffer D, Fisher P, Lucas CP, Dulcan MK, Schwab-Stone ME (2000). NIMH Diagnostic Interview Schedule for Children Version IV (NIMH DISC-IV): description, differences from previous versions, and reliability of some common diagnoses. J Am Acad Child Adolesc Psychiatry 39: 28-38.

Spear LP (2011). Rewards, aversions and affect in adolescence: Emerging convergences across laboratory animal and human data. Dev Cogn Neurosci 1: 390-403.

Steffensen SC, Svingos AL, Pickel VM, Henriksen SJ (1998). Electrophysiological characterization of GABAergic neurons in the ventral tegmental area. J Neurosci 18: 8003-8015.

Tian H, Chen HJ, Cross TH, Edenberg HJ (2005). Alternative splicing and promoter use in the human GABRA2 gene. Brain Res Mol Brain Res 137: 174-183.

Van Leijenhorst L, Zanolie K, Van Meel CS, Westenberg PM, Rombouts SA, Crone EA (2010). What motivates the adolescent? Brain regions mediating reward sensitivity across adolescence. Cereb Cortex 20: 61-69.

Villafuerte S, Heitzeg MM, Foley S, Yau WY, Majczenko K, Zubieta JK et al (2012). Impulsiveness and insula activation during reward anticipation are associated with genetic variants in GABRA2 in a family sample enriched for alcoholism. $\mathrm{Mol}$ Psychiatry 17: 511-519.

Volkow ND, Wang GJ, Maynard L, Fowler JS, Jayne B, Telang F et al (2002). Effects of alcohol detoxification on dopamine D2 receptors in alcoholics: a preliminary study. Psychiatry Res 116: 163-172.

Yau WY, Zubieta JK, Weiland BJ, Samudra PG, Zucker RA, Heitzeg MM (2012). Nucleus accumbens response to incentive stimuli anticipation in children of alcoholics: relationships with precursive behavioral risk and lifetime alcohol use. J Neurosci 32: 2544-2551.

Zucker R, Fitzgerald H, Noll R (1990). Drinking and Drug History (Revised edition, Version 4). University of Michigan Department of Psychiatry, Addiction Research Center: Ann Arbor.

Zucker RA (2006). Alcohol use and alcohol use disorders: a developmental-biopsychosocial systems formulation covering the life course. In: Cicchetti DCohen DJ (eds) Developmental Psychopathology. 2nd edn. Vol Three: Risk, Disorder and Adaptation (Wiley \& Sons: Hoboken, NJ, pp 620-656.

Supplementary Information accompanies the paper on the Neuropsychopharmacology website (http://www.nature.com/npp) 